\title{
DETECCIÓN DE PERÍODOS HÚMEDOS EN LA PROVINCIA DEL CHACO (1961-2005). UNA APROXIMACIÓN A LA IDENTIFICACIÓN DE NÚCLEOS PLUVIOMÉTRICOS.
}

\section{MOISTURE DETECTION PERIODS IN THE PROVINCE OF CHACO (1961-2005). AN APPROACH TO THE IDENTIFICATION OF CORE RAINFALL.}

\author{
Dr. Prof. Oscar Luis Pyszczek. \\ Instituto y Departamento de Geografía \\ Facultad de Humanidades UNNE \\ Docente e investigador del Dpto. de Geografía. \\ Prof. María Emilia Pérez. \\ Instituto y Departamento de Geografía \\ Facultad de Humanidades UNNE \\ Docente e investigadora del Dpto. de Geografía.
}

\section{Resumen}

El presente artículo tiene como objeto de estudio a las precipitaciones acaecidas en la Provincia del Chaco durante el período 1961-2005. Específicamente se ha estudiado tanto a escala anual como mensual, los períodos de meses cuyas precipitaciones hayan sido superiores a los promedios anuales (períodos húmedos). La metodología empleada en la investigación, conforma un aporte original puesto que se ha ensayado un criterio propio para establecer núcleos de precipitaciones superiores a lo normal o núcleos pluviométricos partiendo de la previa implementación del método de quintiles. Estos núcleos pluviométricos han sido definidos como el intervalo de 2 a 5 meses cuyo monto total represente el $50 \%$ o más de los promedios anuales. Los datos estadísticos utilizados provienen de la Red Pluviométrica Provincial registrados por la Administración Provincial del Agua, organismo rector en cuestiones hídricas en la Provincia.

Palabras claves: Precipitaciones; Cuartiles; Períodos Húmedos; Núcleos Pluviométricos.

\begin{abstract}
This article aims to study the rainfall occurred in Chaco Province during the period 1961-2005. Specifically it has been studied both annual and monthly scale, periods of months whose precipitation has been higher than the annual average (wet periods). The methodology used in the research, forms an original contribution as it has tried to establish a criterion cores above normal rainfall or cores based on the previous implementation of the method of precipitation quintiles. These core rainfall have been defined as the interval of 2-5 months from a total amount equal to $50 \%$ or more of annual averages. The statistical data used come from the Red Rainfall recorded by the Provincial Water Administration, the lead agency in water issues in the Province Provincial.
\end{abstract}

Keywords: Rainfall; Quartiles; Humid periods; Pluviometric core.

Publicado en formato digital: Dr. Prof. Oscar Luis Pyszczek. DETECCIÓN DE PERíodos HÚMEDOS EN LA PROVINCIA DEL CHACO (1961-2005). UNA APROXIMACIÓN A LA IDENTIFICACIÓN DE NÚCLEOS PLUVIOMÉTRICOS. Revista Geográfica Digital. IGUNNE. Facultad de Humanidades. UNNE. Año 11. № 22. Julio - Diciembre. 2014. ISSN 1668-5180 Resistencia, Chaco.

En: http://hum.unne.edu.ar/revistas/geoweb/default.htm 


\section{Introducción}

El abordaje de cuestiones vinculadas a los elementos del clima adquiere relevancia en el marco actual de debate global sobre el tan mencionado cambio climático. Al respecto, el presidente de la Organización Meteorológica Mundial (OMM), David Grimes ha declarado: "Lo que observamos actualmente es un incremento en la variabilidad del clima con eventos extremos cada vez más frecuentes, lo cual plantea desafíos a los países de todas las regiones del mundo".(La Nación, 06/04/2013).

El aumento de las frecuencias de tormentas fuertes, granizo, olas de calor, y otros tantos fenómenos meteorológicos y climáticos constituyen una temática de discusión global, como así también los períodos de precipitaciones intensas o períodos de sequía, los cuales conforman eventos extraordinarios o bien extremos.

Los Fenómenos climáticos extremos (fenómenos meteorológicos o climáticos extremos indistintamente) se definen como la ocurrencia de un valor de una variable meteorológica o climática por encima (o por debajo) de un valor de umbral cercano al extremo superior (o inferior) de la horquilla de valores observados de la variable. (IPCC, 2012: 4).

Estos fenómenos no son comunes; por consiguiente, se dispone de pocos datos para evaluar los cambios en su frecuencia o intensidad. Cuanto menos común es el fenómeno, más difícil es determinar los cambios a largo plazo.

En este marco, las precipitaciones que conforman el elemento climático más variable tanto en espacio y tiempo, no queda exenta de ello y reflejan las más minúsculas variaciones de los centros de acción que rigen la circulación atmosférica a escala regional y mundial.

Las precipitaciones ejercen una influencia decisiva en la conformación paisajística y en la valorización del espacio por parte del hombre, ya que no solamente condiciona la supervivencia de las especies, sino que regula su funcionamiento y dinámica. Las lluvias son decisivas para la producción agropecuaria tanto de secano como de riego, el abastecimiento de agua a las ciudades para consumo y producción económica industrial, y en ambientes más extremos, regula el comportamiento social o su interacción, tal es el caso por ejemplo, de las tribus del norte de Níger donde la llegada de las escasas precipitaciones posibilita rituales de cortejo y fertilidad en las sociedades nómades que habitan esa región.

La provincia del Chaco, por su definición económica de carácter eminentemente agropecuario, y el predominio de la técnica de secano, late al ritmo de las precipitaciones. Su escasez como su abundancia condicionan las actividades económicas predominantes.

A sabiendas de ello, el presente trabajo intenta convertirse en un aporte al conocimiento del vital elemento, proyectando tendencias y previendo sus fluctuaciones a lo largo del tiempo y del territorio provincial.

El estudio de los meses lluviosos o muy lluviosos como también el de los períodos húmedos, en tanto su intensidad y extensión espacio- temporal no ha constituido temática habitual en la investigación regional y mucho menos en la determinación de criterios de definición específicos. Es por ello que la presente investigación constituye un ensayo de criterio tendiente a la determinación de núcleos pluviométricos, que responden a incremento de actividades atmosféricas insertas en la variación normal de los elementos climáticos alrededor de sus valores medios.

Las estaciones pluviométricas de referencia han sido cinco: Resistencia, Machagai, San Bernardo, Los Frentones y Juan José Castelli, las cuales han sido seleccionadas en base a la existencia de datos para la serie contemplada y por su distribución en el territorio provincial.

Las instancias metodológicas han sido fundamentalmente tres: en primer lugar se han normalizado los datos estadísticos correspondientes los puestos pluviométricos seleccionados en forma gráfica cartográfica; posteriormente se ha implementado el método de quintiles a la serie estadística para determinar los meses lluviosos y muy lluviosos. Finalmente se ha delimitado los criterios y umbrales para identificar los períodos pluviométricos y luego representarlos visualmente en las series mensuales cada una de los puestos pluviométricos seleccionados.

\section{Localización de los puestos pluviométricos seleccionados en la investigación.}

Los datos suministrados por la Administración Provincial del Agua (A.P.A.), consisten en montos pluviométricos mensuales para la serie de años comprendidos entre 1961-2005, de los cuales se han seleccionado los registros correspondientes a cinco localidades, distribuidas en todo el territorio

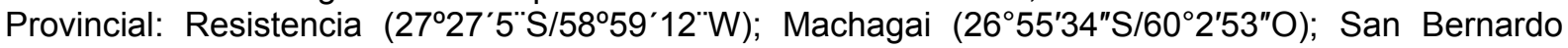

Publicado en formato digital: Dr. Prof. Oscar Luis Pyszczek. DETECCIÓN DE PERíodos HÚMEDOS EN LA PROVINCIA DEL CHACO (1961-2005). UNA APROXIMACIÓN A LA IDENTIFICACIÓN DE NÚCLEOS PLUVIOMÉTRICOS. Revista Geográfica Digital. IGUNNE. Facultad de Humanidades. UNNE. Año 11. № 22. Julio - Diciembre. 2014. ISSN 1668-5180 Resistencia, Chaco.

En: http://hum.unne.edu.ar/revistas/geoweb/default.htm 
Revista Geográfica Digital. IGUNNE. Facultad de Humanidades. UNNE. Año 11. № 22. Julio - Diciembre 2014. ISSN 1668-5180 Resistencia, Chaco

$\left(27^{\circ} 17^{\prime} 12^{\prime \prime} \mathrm{S} / 60^{\circ} 42^{\prime} 46^{\prime \prime O}\right)$; Juan José Castelli $\left(25^{\circ} 56^{\prime} 48^{\prime \prime} \mathrm{S} / 60^{\circ} 37^{\prime} 12^{\prime \prime O}\right)$; Los Frentones $\left(26^{\circ} 24^{\prime \prime} 25^{\prime \prime} \mathrm{S} / 61^{\circ} 24^{\prime} 47^{\prime \prime} \mathrm{O}\right)$.

Fig. 1. Puestos Pluviométricos de Referencia.

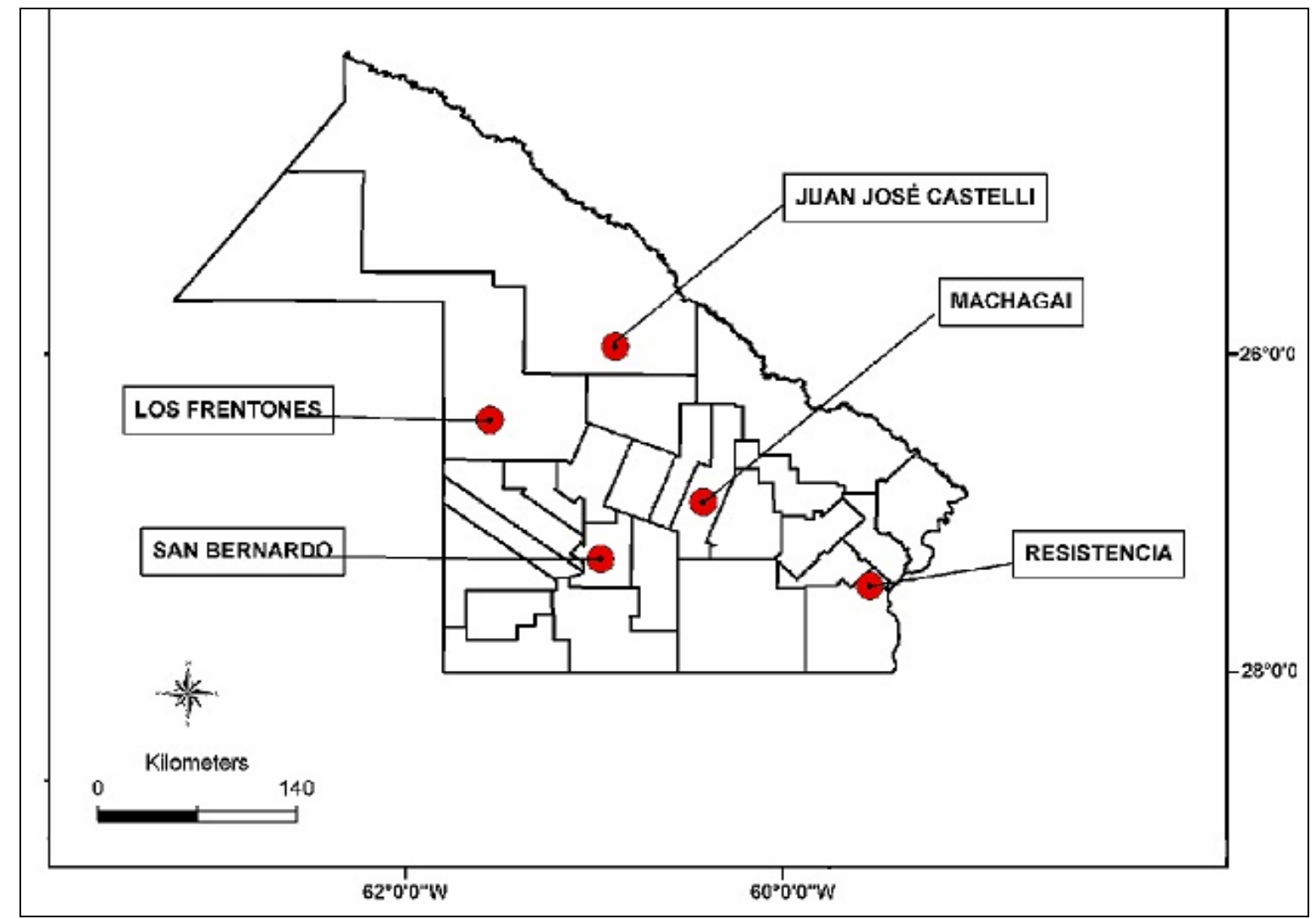

Fuente: elaboración propia.

La Figura $N^{\circ} 1$, muestra la localización de los puestos pluviométricos seleccionados. Los mismos se encuentran distribuidos a lo ancho del territorio provincial, posibilitando mediante su ubicación, la detección de las diferencias temporo-espaciales en cuanto a las manifestaciones pluviométricas. Los criterios contemplados para su selección tuvieron un carácter combinado: en primer lugar, se observó la longitud de las series de registros pluviales de cada una de los puestos, quedando fuera del período en estudio, aquellos ubicados en el noroeste del Chaco, puesto que sus registros son recientes.

Por otro lado se valoró la ubicación en el territorio provincial de cada una de las 64 estaciones y puestos existentes. La Red Pluviométrica de la provincia del Chaco (Fig. $\boldsymbol{N}^{\circ}$ 2), presenta la particularidad de que cada una de sus estaciones de registros se sitúan muy cercanas entre sí, y a su vez, están concentradas en las localidades emplazadas sobre las principales vías de comunicación provincial. Los puestos pluviométricos escogidos para la investigación están instalados a distancias considerables unos de otros de manera tal, poder avizorar las variaciones espaciales de los montos. Luego de seleccionar y localizar los puestos pluviométricos, se inició la etapa de tratamiento y normalización de los datos estadísticos.

La circunstancia que condicionó la investigación al período 1961-2005; fue el hecho de que la localidad de San Bernardo, cuenta con registros pluviométricos sólo a partir del año 1961, por lo tanto a fines comparativos entre los puestos pluviométricos seleccionados es que se delimito dicho período.

Publicado en formato digital: Dr. Prof. Oscar Luis Pyszczek. DETECCIÓN DE PERíodos HÚMEDOS EN LA PROVINCIA DEL CHACO (1961-2005). UNA APROXIMACIÓN A LA IDENTIFICACIÓN DE NÚCLEOS PLUVIOMÉTRICOS. Revista Geográfica Digital. IGUNNE. Facultad de Humanidades. UNNE. Año 11. № 22. Julio - Diciembre. 2014. ISSN 1668-5180 Resistencia, Chaco.

En: http://hum.unne.edu.ar/revistas/geoweb/default.htm 
Fig. No 2. Red Pluviométrica de la Provincia del Chaco.

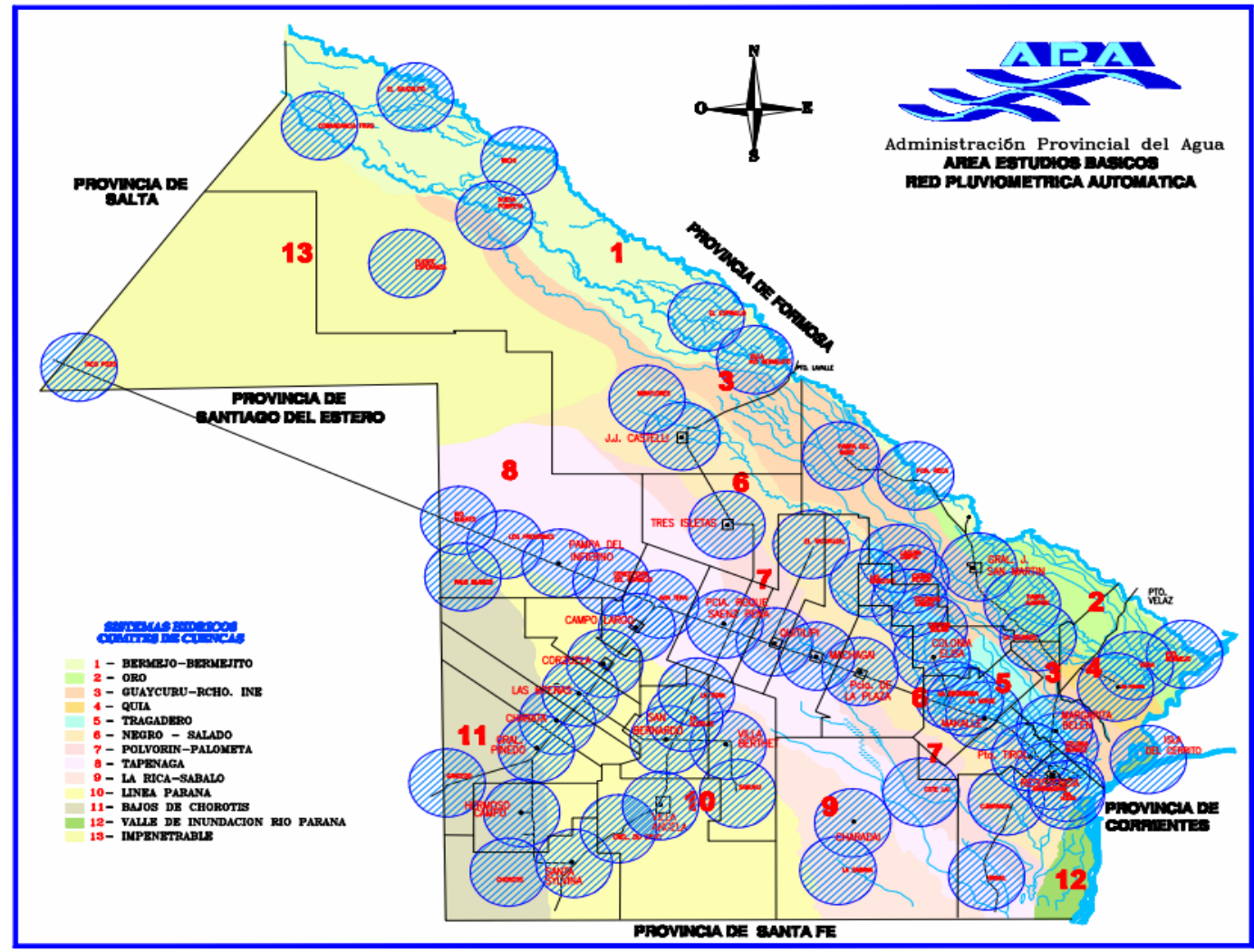

Fuente: Administración Provincial del Agua.

\section{Distribución temporal de las precipitaciones según puesto pluviométrico.}

Las precipitaciones en la provincia, presentan una disminución en sentido este - oeste, reflejándose en los montos pluviométricos promedios de cada uno de los puestos que siguen este mismo componente de orientación. No obstante, su ritmo interanual es mucho más contrastado dependiendo de controles macros-atmosféricos, como por ejemplo las temperaturas de los océanos entre los más importantes.

La Figura $N^{\circ} 3$, representa la distribución de las precipitaciones medias anuales, según puestos pluviométricos, desde el año 1961 al 2005.

En Resistencia, los montos pluviométricos siempre han superado la barrera de los $1000 \mathrm{~mm}$ anuales lo que conforma un rasgo constante en los 45 años de la serie a excepción del año 1989, que se alza con el menor monto pluviométrico del intervalo en estudio $(920 \mathrm{~mm}$.).

Para la localidad de Machagai, se puede observar un mayor contraste pluviométrico, con un incremento del agrupamiento de los años más lluviosos como en el trienio 1966-68; y 1973-76, intercalados con períodos de menores precipitaciones, tales como: 1963-65; 1969-71 y 1977-79.

La serie estadística para San Bernardo, no evidencia mayores diferencias de las enunciadas para las anteriores estaciones, no obstante puede distinguirse la disminución en los valores pluviométricos absolutos acaecidos en este lugar. El valor de $1200 \mathrm{~mm}$, define los montos pluviométricos más importantes, en los cuales tan solo11 años lo superan, registrándose en el trienio 1985-87 un pico en los datos con montos todos ellos superiores a $1400 \mathrm{~mm}$.

La localidad de Juan José Castelli, presenta precipitaciones máximas que exceden los $1000 \mathrm{~mm}$ anuales, y abarca 9 (nueve) años específicos: 1967,1968,1980,1981,1985,1986,1991,2000 y 2002. Los montos de los años mencionados, conforman registros excepcionales alejados del promedio pluvial que le corresponde para el puesto de observación.

Por último la localidad de los Frentones ubicada denota una tendencia particular, donde el año 1988, divide a la serie en dos partes, pues es en este año en donde se registra el menor monto de la serie y actúa como un quiebre en el régimen de datos.

Publicado en formato digital: Dr. Prof. Oscar Luis Pyszczek. DETECCIÓN DE PERÍOdos HÚMEDOS EN LA PROVINCIA DEL CHACO (1961-2005). UNA APROXIMACIÓN A LA IDENTIFICACIÓN DE NÚCLEOS PLUVIOMÉTRICOS. Revista Geográfica Digital. IGUNNE. Facultad de Humanidades. UNNE. Año 11. Nº 22. Julio - Diciembre. 2014. ISSN 1668-5180 Resistencia, Chaco.

En: http://hum.unne.edu.ar/revistas/geoweb/default.htm 
Revista Geográfica Digital. IGUNNE. Facultad de Humanidades. UNNE. Año 11. № 22.

Julio - Diciembre 2014. ISSN 1668-5180 Resistencia, Chaco

Fig. № 3. Precipitaciones medias anuales según puestos pluviométricos. 1961 al 2005.
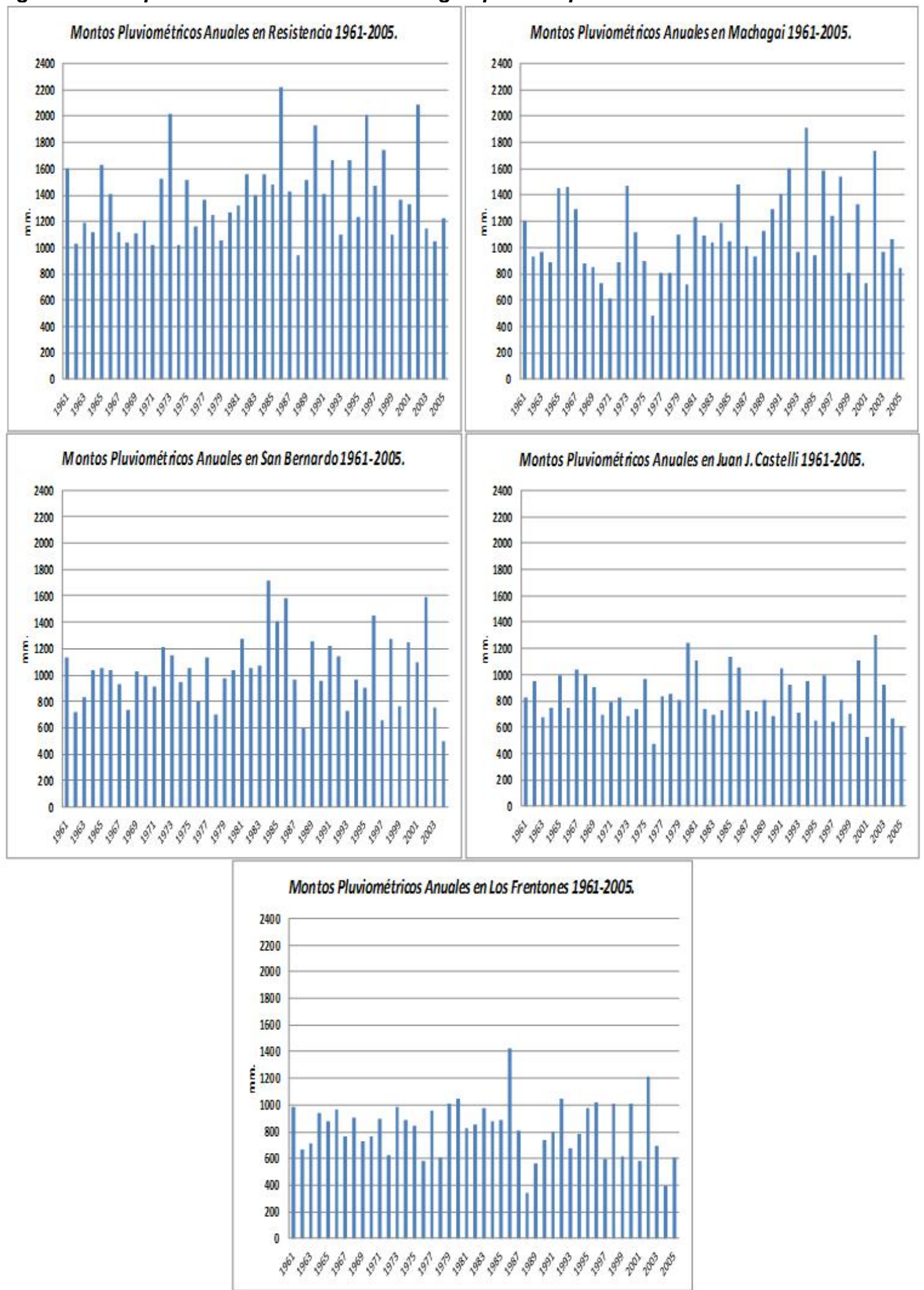

Fuente: elaboración propia en base a los datos de la Administración Provincial del Agua.

Publicado en formato digital: Dr. Prof. Oscar Luis Pyszczek. DETECCIÓN DE PERÍODOS HÚMEDOS EN LA PROVINCIA DEL CHACO (1961-2005). UNA APROXIMACIÓN A LA IDENTIFICACIÓN DE NÚCLEOS PLUVIOMÉTRICOS. Revista Geográfica Digital. IGUNNE. Facultad de Humanidades. UNNE. Año 11. № 22. Julio - Diciembre. 2014. ISSN 1668-5180 Resistencia, Chaco.

En: http://hum.unne.edu.ar/revistas/geoweb/default.htm 


\section{Determinación de meses lluviosos y muy lluviosos según método de Quintiles.}

Uno de los métodos utilizados en el campo de la Geografía Física para determinar meses o períodos secos y húmedos, es el de los Cuartiles o Cuartilas, como así también el de los Quintiles y Percentiles, los cuales siguen procesos estadísticos similares.

Como consecuencia de que sus características y resultados se adecuan a las necesidades del trabajo, en esta oportunidad, se ha optado por implementar el método de Quintiles. Este método permite dividir a la serie estadística de acuerdo a la gradación de valores y determinar los meses húmedos o lluviosos. Éste método de detección es utilizado por varios Institutos y Servicios Nacionales de Meteorología (como por ejemplo el de Argentina y el de España), permitiendo clasificar los años o meses en: muy secos, secos, normales, lluviosos y muy lluviosos en función de la precipitación anual y/o mensual:

\begin{tabular}{ll}
\hline Año muy seco & $P<Q_{1}$ \\
Año seco & $Q_{1} \leq P<Q_{2}$ \\
Año normal & $Q_{2} \leq P<Q_{3}$ \\
Año lluvioso & $Q_{3} \leq P<Q_{4}$ \\
Año muy lluvioso & $P>Q_{4}$ \\
\hline
\end{tabular}

El método por Quintiles "permite distinguir cuatro quintiles que dividen la serie en cinco subconjuntos de igual tamaño" (Fernández García, 1995), de la siguiente manera:

$>$ Q1: el $20 \%$ de las observaciones menores al primer quintil y el $80 \%$ superior a este.

> Q4: el $20 \%$ de las observaciones mayores al cuarto quintil y el $80 \%$ inferior a este.

$>$ Q2: el $40 \%$ de las observaciones menores al segundo quintil y el $60 \%$ de las observaciones superior a este.

$>$ Q3: el $40 \%$ de las observaciones superiores al tercer quintil y el $60 \%$ de las observaciones inferiores a este.

Para llevar a cabo la división de los datos, en primer lugar es necesario ordenar los valores de menor a mayor.

La definición de los quintiles se realizó a través de la formula:

$$
i=\underline{(2 n \cdot c)+1}
$$

2

Donde:

$\mathrm{i}$ = Rango del elemento o lugar que ocupa según su valor;

$\mathrm{n}=$ total de valores de la muestra;

$\mathrm{c}=$ Frecuencia Relativa de la cuartila.

El resultado final de la implementación de la metodología puede observarse en las Tablas 2 a 6 , correspondientes a la identificación de los meses lluviosos, muy lluviosos y núcleos pluviométricos, para la serie de años contemplados en la investigación.

En Resistencia, Desde 1961 a 1971, solo existe un año que supera los seis meses lluvioso o muy lluvioso, 1965, el cual está dentro de la categoría de año muy lluvioso según se expone en ítem anterior. Desde 1972-1979, existen una serie de años que alcanzan la frecuencia 6: los de mayor frecuencia son 1972 y 1973, los cuales tienen una frecuencia de meses lluviosos equivalentes a 9, luego 1975-1977-1978 seguido de un período donde bajan los valores de frecuencia, hasta el trienio1984-1986, en donde se registra nuevamente un pico de frecuencias. Otro período importante

Publicado en formato digital: Dr. Prof. Oscar Luis Pyszczek. DETECCIÓN DE PERíodos HÚMEDOS EN LA PROVINCIA DEL CHACO (1961-2005). UNA APROXIMACIÓN A LA IDENTIFICACIÓN DE NÚCLEOS PLUVIOMÉTRICOS. Revista Geográfica Digital. IGUNNE. Facultad de Humanidades. UNNE. Año 11. Nº 22. Julio - Diciembre. 2014. ISSN 1668-5180 Resistencia, Chaco.

En: http://hum.unne.edu.ar/revistas/geoweb/default.htm 
se registra entre los años 1989-1997, intercalado frecuencias elevadas, con otros por debajo de la frecuencia 6. Finalmente en el comienzo del siglo XXI y en su primer lustro solamente se registra el año 2002 con una frecuencia de 7 meses. Se observa además, que entre los años 1961-1982 existe una concentración de los meses muy lluviosos en el período invernal. A partir del año 1982, se registran los meses lluviosos en las estaciones de otoño y primavera.

En Machagai, La serie comienza con el año 1961 donde hay seis meses lluviosos, seguidos de un lapso donde la frecuencia es más baja. En el trienio 1965-1967 nuevamente existe un repunte, para posteriormente mermar. Los años 1973-1974, junto con el año 1979, son los únicos de toda la década de 1970 superan la frecuencia 6. La década de 1980 se presenta, con una tendencia similar comenzando en los años 1981-82 con frecuencias de 6 meses lluviosos, seguido del año 1986 que posee la segunda mayor frecuencia de meses lluviosos, el cual llega a 8 meses. En la década de 1990 se perfila con mayor números de años con frecuencias de 6 o más como por ejemplo el trienio 1990-92,1994, el cual posee una frecuencia de 9 meses, es decir el $75 \%$ del año con lluvias superiores a lo normal. Finalizando en el trienio 1996-98 con sendos frecuencias lluviosas y muy lluviosas. Los años 2000 y 2003 a su vez presentan frecuencia de 6 meses.

San Bernardo presenta dos períodos definidos: el primero abarca los año 1961-1972-1973-1977-1979 con frecuencias que van desde 6 meses a 7 meses. El segundo período, comienza con cuatro años 1984-1987- que poseen alta frecuencia, siendo 1984 el año que presenta 9 meses lluviosos y muy lluviosos.

Los años 1988 y 1997 son dos "quiebres" en el gráfico, pues presentan una frecuencia de 1 mes siendo las menores de toda la serie representada. Entre finales de la década de 1990 y principios de los 2000 se observa una mayor recurrencia de años con frecuencias altas siendo 2002 el año con mayor frecuencia.

En Castelli, la distribución en la serie estadística de los meses lluviosos y muy lluviosos, arroja que desde los años 1960-1980 se evidencia un agrupamiento en los meses de invierno y a partir de 1980 ya comienzan a sucederse en estaciones intermedias.

Finalmente en los Frentones, la distribución de los meses en la serie estadística presenta un ritmo similar al registrado en Castelli, donde en los años 1960-1980 se evidencia un agrupamiento en los meses de invierno y a partir de 1980 ya comienzan a sucederse en estaciones intermedias.

\section{Aproximación a la determinación de los Períodos Húmedos o núcleos pluviométricos.}

El problema fundamental al momento de definir y determinar los períodos de alta pluviosidad, es el hecho de que no existe un criterio formal o estándar para identificar a los mismos. Si bien los métodos matemáticos conforman una alternativa valedera para su estudio, presentan algunas dificultades en sus cálculos, como por ejemplo, el hecho de no tener en cuenta el ritmo de precipitaciones y sólo basarse en los valores estadísticos. El "vacío" de parámetros en los cuales respaldarnos (valores numéricos, umbrales), que nos permitan identificar estos intervalos conforman el principal problema acaecido, lo que ha llevado a generar un criterio, que sea expresión genuina y no forzada de los datos.

Por todo lo mencionado, se han ensayado varias alternativas y parámetros en la determinación de los períodos húmedos o núcleos pluviométricos, a fin de generar un criterio que incluya integralmente a las cuestiones inherentes del elemento en estudio y lograr en última instancia un complemento con otros métodos como por ejemplo el de los Quintiles. Si bien se consideran varios aspectos metodológicos trabajados en diferentes estudios referidos a la temática, se ha procurado esbozar una técnica particular adaptada a las condiciones climáticas singulares del Chaco y que sea expresión fehaciente y real del comportamiento pluvial en nuestra provincia.

Al hablar de períodos de elevada pluviosidad estamos vinculando un lapso determinado de tiempo, con precipitaciones abundantes o por lo menos por encima de los montos normales, las cuales imprimirán sus efectos en los sistemas ecológicos y productivos del espacio geográfico.

De acuerdo a lo mencionado, se considera como período húmedo, a los lapsos temporales donde las precipitaciones superan ampliamente a los valores medios. Este concepto surge más bien como antónimo de lo definido como período seco, pues existe una amplia selección bibliográfica que se

Publicado en formato digital: Dr. Prof. Oscar Luis Pyszczek. DETECCIÓN DE PERíodos HÚMEDOS EN LA PROVINCIA DEL CHACO (1961-2005). UNA APROXIMACIÓN A LA IDENTIFICACIÓN DE NÚCLEOS PLUVIOMÉTRICOS. Revista Geográfica Digital. IGUNNE. Facultad de Humanidades. UNNE. Año 11. № 22. Julio - Diciembre. 2014. ISSN 1668-5180 Resistencia, Chaco.

En: http://hum.unne.edu.ar/revistas/geoweb/default.htm 
ocupa de los lapsos deficitarios de precipitaciones. Para comenzar esta elaboración, se ha considerado la identificación de los meses lluviosos y muy lluviosos mediante el método de quintiles explicado anteriormente.

Las precipitaciones constituyen el elemento climático, con mayor variabilidad en cuanto a montos pluviométricos se refiere, tanto en el orden mensual como anual. De allí surge la necesidad de tener un valor estándar que represente a la serie de datos para cada una de las estaciones meteorológicas en estudio. Para la presente elaboración se ha optado por tomar como valor estándar o de referencia a los promedios de los montos pluviométricos anuales, para cada una de los puestos. Si bien los valores promedios tienen la características de enmascarar ciertos oscilaciones estadísticas que pueden ser significativas, el objetivo definido es identificar los períodos húmedos y no el régimen pluviométrico.

Tabla No1: Promedios pluviométricos anuales 1961-2005 utilizados como valores de referencia.

\begin{tabular}{|l|c|}
\hline Puestos Pluviométricos & Montos pluviométricos promedios (mm) \\
\hline RESISTENCIA & 1391,2 \\
\hline MACHAGAI & 1104,5 \\
\hline SAN BERNARDO & 1036,2 \\
\hline J. J. CASTELLI & 839,5 \\
\hline LOS FRENTONES & 825,0 \\
\hline
\end{tabular}

Una vez establecidos los valores de referencia con los cuales se trabajará, se procedió a determinar el criterio que nos permita identificar períodos húmedos.

Si consideramos los meses lluviosos y muy lluviosos (obtenidos mediante el método matemático de quintiles), permitirá definir el lapso de tiempo para considerar los períodos húmedos.

Al conformar intervalos en donde se presenta un exceso de precipitaciones; la concatenación de los meses considerados húmedos y muy húmedos constituyen lapsos de abundancia pluvial; pero para que ese exceso de humedad provocado por las lluvias no pase inadvertido, es necesario que precipite en un intervalo consecutivo, de modo tal se advierta dicho exceso. Esto constituye lo que llamaremos Núcleos Pluviales o Pluviométricos.

La estructuración de períodos húmedos a través de núcleos donde las lluvias se manifiestan de modo considerable permitió arribar al siguiente criterio:

"Núcleo Pluviométrico será, aquel lapso de 2 a 5 meses considerados húmedos que de modo consecutivo, concentren $50 \%$ o más del monto pluviométrico anual".

Para elaborar este criterio, se ha considerado lo siguiente:

a. En primer lugar, los meses lluviosos y muy lluviosos son los que constituyen los núcleos húmedos, o pluviométricos.

b. Las precipitaciones deben ser significativas en un lapso relativamente corto de tiempo; es sabido que las precipitaciones son muy variables tanto en un lapso mensual como anual, no obstante, desde el punto de vista matemático si el $50 \%$ de las lluvias se producen en 6 meses (50\% del año), tiende a asemejarse a un comportamiento promedio, si las precipitaciones se distribuyeran homogéneamente durante todo el año ( 6 meses $=50 \%$ del año). Es por ello que, 5 meses o menos que concentren $50 \%$ o más del monto pluviométrico de referencia, constituiría la base para la determinación de los núcleos pluviales.

c. En la determinación de los núcleos pluviométricos se utilizaron en esta oportunidad los montos pluviométricos anuales. Es necesario tener en cuenta que la suma de los montos deben superar el $50 \%$ o más del volumen pluviométrico anual.

Publicado en formato digital: Dr. Prof. Oscar Luis Pyszczek. DETECCIÓN DE PERíOdos HÚMEDOS EN LA PROVINCIA DEL CHACO (1961-2005). UNA APROXIMACIÓN A LA IDENTIFICACIÓN DE NÚCLEOS PLUVIOMÉtRICOS. Revista Geográfica Digital. IGUNNE. Facultad de Humanidades. UNNE. Año 11. N 22. Julio - Diciembre. 2014. ISSN 1668-5180 Resistencia, Chaco.

En: http://hum.unne.edu.ar/revistas/geoweb/default.htm 
Revista Geográfica Digital. IGUNNE. Facultad de Humanidades. UNNE. Año 11. № 22.

Julio - Diciembre 2014. ISSN 1668-5180 Resistencia, Chaco

La distribución de los núcleos pluviométricos se observan las tablas $N^{\circ} 2-3-4-5-6$, para cada uno de los puestos seleccionados.

\section{Análisis espacio-temporal de los períodos húmedos.}

La aplicación de este criterio en la serie estadística, generó particularidades para cada una de los puestos pluviométricos tomados como referencia:

En Resistencia se aprecia (Tabla N²) que los períodos húmedos se encuentran localizados en los siguientes años:

En 1965 se inicia en el mes de agosto y se prolonga hasta diciembre, con 5 meses de duración que concentran el $53 \%$ del monto anual, continuándose de enero a marzo del año 1966 acumulando un total de $869 \mathrm{~mm}$ que representan el 52\% del volumen anual para ese año. El bienio 1972-1973 constituye un período lluvioso prolongado.

Desde el año 1973, se extiende un intervalo que comprende 10 años, en donde se registran meses lluviosos y muy lluviosos pero sin constituirse períodos húmedos.

Entre los años 1984-85-86, se detectan períodos húmedos en el primer semestre de cada uno de los años, (en 19843 meses, 1985 de 4 meses y 1986 de 5 meses), todos superando el $50 \%$ de los montos pluviométricos anuales.

A partir de allí los períodos húmedos se sucedieron de manera intercalada y ya no asociada entre sí. Podemos mencionar como tales, los años 1992; principios de 1995; finales de 1996; en el año 1998 año de las recordadas inundaciones en la provincia del Chaco, se evidencia un período húmedo que comienza con meses lluviosos a finales de 1997 y se constituye núcleos pluviales en los primeros 4 meses de 1998 (enero a abril).

Luego se observa que en dos meses de 2002 (enero y abril) precipitan $1146 \mathrm{~mm}$ que constituye el $54 \%$ del monto pluviométrico anual.

En Machagai (Tabla N³) se observa núcleos pluviométricos en el bienio 1965 (meses de octubre, noviembre y diciembre) y enero, febrero y marzo de 1966, repitiéndose en los años 1973-74. Esta tendencia constituye una constante en el resto de las estaciones de Referencia (San Bernardo, Castelli y Los Frentones), los cuales, con algunas variaciones particulares, denotan mayor actividad atmosférica.

Machagai presenta entre los años 1983-86 núcleos caracterizados por el breve espacio de tiempo (2 meses), excepto en el otoño-invierno de 1986, donde se observa un núcleo pluvial de 5 meses. También se detectan núcleos Pluviales para los años 1991; 1994 a 1997 de forma consecutiva, donde se destaca el año 1994 en donde se localizan dos períodos Pluviales en el mismo año. Finalizando con dos períodos intercalados en el año 2000 y 2004.

En San Bernardo (Tabla N4), se observa períodos húmedos en los años 1961-1965/66-1972/73$1980 / 81$ y en el trienio 1984-86 con períodos importantes de precipitaciones en especial 1986, en donde se registra dos núcleos en el mismo año. Luego se distribuyen de modo intercalado (19891991-1992/93) hasta el bienio 2001-02 en donde se evidencia mayor presencia pluvial.

En Juan José Castelli y de Los Frentones (Tablas $N^{\circ} 5$ y 6), los núcleos pluviométricos son mucho más espaciados que el resto de las estaciones de la provincia, evidenciándose dicha variabilidad y distribución por tratarse de puestos situados más hacia el oeste, en donde las masas de aire presentan una disminución marcada de la humedad que poseen y la ocurrencia de dichos núcleos pluviales dependerá de la intensidad (Constancia temporal) de la masa de aire, como así también de la humedad que contenga la misma.

Es así como la estación de Castelli presenta períodos húmedos en el año 1975, coincidiendo con los Frentones en donde se registra también períodos en el bienio 1974-75. Estos años parecen constituir un retraso a los núcleos que se registran en los años 1972-1973 en el resto de las estaciones meteorológicas.

En el caso de Castelli, los años 1978, 1980 y 1986 presentan doble núcleo pluviométrico que se suceden en cada uno de dichos años. Luego se dan períodos en los años 1991-1996- 1998-2000 y 2002 este último coincidiendo con los datos de otros puestos descritos.

La estación de los Frentones, presenta un período húmedo importante en el trienio 1978-80, con importantes montos pluviométricos caídos.

Es evidente como el comportamiento pluvial determina que los principales núcleos pluviométricos se desarrollen en la estación estival, los cuales son más importantes, mientras que los núcleos

Publicado en formato digital: Dr. Prof. Oscar Luis Pyszczek. DETECCIÓN DE PERÍODOS HÚMEDOS EN LA PROVINCIA DEL CHACO (1961-2005). UNA APROXIMACIÓN A LA IDENTIFICACIÓN DE NÚCLEOS PLUVIOMÉTRICOS. Revista Geográfica Digital. IGUNNE. Facultad de Humanidades. UNNE. Año 11. No 22. Julio - Diciembre. 2014. ISSN 1668-5180 Resistencia, Chaco.

En: http://hum.unne.edu.ar/revistas/geoweb/default.htm 
pluviométricos más cortos se dan en otoño. Al igual que otras estaciones, para el año 1986, se presentan dos núcleos pluviales. Luego sigue un período más espaciado en donde se suscitan núcleos de distintas duraciones, en donde se destacan los años 1992 y 2002 en cuanto a la extensión de los núcleos.

Publicado en formato digital: Dr. Prof. Oscar Luis Pyszczek. DETECCIÓN DE PERíodos HÚMEDOS EN LA PROVINCIA DEL CHACO (1961-2005). UNA APROXIMACIÓN A LA IDENTIFICACIÓN DE NÚCLEOS PLUVIOMÉtRICOS. Revista Geográfica Digital. IGUNNE. Facultad de Humanidades. UNNE. Año 11. N 22. Julio - Diciembre. 2014. ISSN 1668-5180 Resistencia, Chaco.

En: http://hum.unne.edu.ar/revistas/geoweb/default.htm 
Celeste: mes lluvioso.

Azul: mes muy lluvioso.

Grisado Naranja: Núcleos

pluviométricos.

Tabla No 2. Meses lluviosos y Núcleos pluviométricos en Resistencia. 1961-2005.

\begin{tabular}{|c|c|c|c|c|c|c|c|c|c|c|c|c|}
\hline AÑO & $\overline{\bar{E}}$ & $F$ & 11 & A & $M$ & J & $J$ & A & $\mathrm{s}$ & 0 & $\mathrm{~N}$ & $D$ \\
\hline 1961 & 899 & 2008 & 320 & 100 & 80 & 30 & 20 & 40 & 20 & 180 & 320 & 90 \\
\hline 1962 & 210 & 40 & 148 & 220 & 90 & 3 & 20 & 20 & 20 & 80 & 120 & 60 \\
\hline 1963 & 200 & 120 & 94 & 140 & 80 & 20 & 40 & 5 & 180 & 70 & 100 & 140 \\
\hline 1964 & 90 & 120 & 200 & 216 & 20 & 30 & 4 & 21 & 81 & 54 & 75 & 208 \\
\hline 1965 & 103 & 182 & 44 & 241 & 88 & 70 & 29 & 56 & $8 \pi$ & 234 & $2 \sqrt{213}$ & 285 \\
\hline 1966 & 378 & 165 & 199 & 150 & 64 & 7 & 43 & 16 & 51 & 105 & 105 & 130 \\
\hline 1967 & 173 & 241 & 180 & 50 & 38 & 22 & 71 & 52 & 59 & 114 & 107 & 11 \\
\hline 1968 & 140 & 109 & 31 & 38 & 38 & 78 & 76 & 152 & 60 & 152 & 86 & 78 \\
\hline 1969 & 294 & 130 & 48 & 105 & 144 & 10 & 20 & 19 & 60 & 117 & 127 & 34 \\
\hline 1970 & 123 & 208 & 112 & 31 & 84 & 46 & 44 & 64 & 215 & 104 & 76 & 97 \\
\hline 1971 & 151 & 69 & 190 & 101 & 101 & 12 & 58 & 70 & 34 & 61 & 41 & 133 \\
\hline 1972 & 199 & 68 & 203 & 182 & 92 & 95 & 88 & 68 & 32 & 110 & 243 & 1558 \\
\hline 1973 & 1541 & 143 & 256 & 189 & 153 & 100 & 102 & 85 & 38 & 137 & 105 & 266 \\
\hline 1974 & 121 & 192 & 70 & 83 & 163 & 23 & 67 & 51 & 27 & 38 & 46 & 145 \\
\hline 1975 & 44 & 158 & 376 & 180 & 59 & 68 & 34 & 109 & 89 & 71 & 203 & 128 \\
\hline 1976 & 266 & 104 & 222 & 65 & 30 & 16 & 30 & 18 & 35 & 132 & 96 & 148 \\
\hline 1977 & 246 & 105 & 117 & 98 & 140 & 21 & 52 & 58 & 13 & 87 & 233 & 193 \\
\hline 1978 & 149 & 130 & 35 & 56 & 106 & 69 & 86 & 72 & 49 & 150 & 200 & 152 \\
\hline 1979 & 50 & 81 & 140 & 81 & 6 & 65 & 29 & 64 & 58 & 135 & 104 & 242 \\
\hline 1980 & 92 & 79 & 166 & 114 & 114 & 90 & 10 & 60 & 60 & 154 & 237 & 95 \\
\hline 1981 & 144 & 233 & 198 & 108 & 129 & 76 & 45 & 20 & 29 & 107 & 171 & 57 \\
\hline 1982 & 82 & 135 & 72 & 83 & 95 & 267 & 22 & 116 & 167 & 29 & 368 & 126 \\
\hline 1983 & 296 & 142 & 59 & 422 & 203 & 7 & 75 & 2 & 0 & 70 & 80 & 44 \\
\hline 1984 & 248 & 47 & 283 & 142 & 157 & 132 & 7 & 11 & 81 & 135 & 139 & 50 \\
\hline 1985 & 72 & 276 & 235 & 321 & 1007 & 39 & 65 & 52 & 108 & 49 & 20 & 83 \\
\hline 1986 & 72 & 122 & 465 & 5) & 124 & 166 & 46 & 29 & 176 & 181 & 217 & 89 \\
\hline 1987 & 177 & 269 & 42 & 242 & 66 & 45 & 132 & 41 & 26 & 56 & 201 & 132 \\
\hline 1988 & 189 & 76 & 93 & 148 & 5 & 41 & 2 & 45 & 67 & 119 & 92 & 64 \\
\hline 1989 & 70 & 108 & 272 & 293 & 4 & 50 & 70 & 99 & 107 & 131 & 118 & 191 \\
\hline 1990 & 130 & 173 & 136 & 528 & 60 & 71 & 7 & 69 & 51 & 359 & 125 & 224 \\
\hline 1991 & 96 & 167 & 23 & 175 & 248 & 53 & 18 & 3 & 56 & 108 & 180 & 286 \\
\hline 1992 & 107 & 1233 & 293 & 229 & 27 & 99 & 42 & 53 & 69 & 234 & 119 & 168 \\
\hline 1993 & 237 & 14 & 177 & 64 & 43 & 59 & 17 & 20 & 60 & 109 & 259 & 44 \\
\hline 1994 & 195 & $\begin{array}{r}149 \\
\end{array}$ & 362 & 121 & $\frac{454}{134}$ & 91 & 34 & 56 & 59 & 155 & 205 & 104 \\
\hline 1995 & 2221 & 368 & 232 & 57 & 79 & 5 & 32 & 32 & 16 & 63 & 79 & 100 \\
\hline 1996 & 256 & 326 & 142 & 411 & 14 & 3 & 7 & 47 & 100 & 194 & 457 & 320 \\
\hline 1997 & 164 & 474 & 12 & 68 & 79 & 64 & 27 & 23 & 42 & 23 & 276 & 210 \\
\hline 1998 & 1232 & 369 & 212 & 398 & 38 & 60 & 7 & 43 & 32 & 76 & 160 & 174 \\
\hline 1999 & 130 & 153 & 296 & 106 & 108 & 34 & 39 & 0 & 17 & 66 & 80 & 72 \\
\hline 2000 & 72 & 220 & 72 & 129 & 93 & 60 & 4 & 0 & 27 & 216 & 166 & 308 \\
\hline 2001 & 182 & 130 & 177 & 154 & 10 & 141 & 20 & 46 & 70 & 156 & 197 & 49 \\
\hline 2002 & 274 & 123 & 382 & 1864 & 87 & 44 & 87 & 31 & 65 & 151 & 176 & 303 \\
\hline 2003 & 68 & 106 & 118 & 114 & 2 & 11 & 60 & 63 & 22 & 137 & 164 & 282 \\
\hline 2004 & 35 & 42 & 81 & 146 & 2 & 55 & 22 & 20 & 101 & 197 & 204 & 145 \\
\hline 2005 & 69 & 86 & 142 & 347 & 87 & 67 & 4 & 19 & 48 & 67 & 189 & 95 \\
\hline
\end{tabular}

Publicado en formato digital: Dr Prof. Oscar Luis Pyszczek. DETECCION DE PERIOODOS HÚMEDOS EN LA PROVINCIA DEL CHACO (1961-2005), UNA APROXIMACIÓN A LA IDENTIFICACIÓN DE NÚCLEOS PLUVIOMÉTRICOS. Revista Geográfica Digital. IGUNNE. Facultad de Humanidades. UNNE. Año 11. No 22. Julio - Diciembre. 2014. ISSN 1668-5180 Resistencia, Chaco.

En: http://hum.unne.edu.ar/revistas/geoweb/default.htm 
Celeste: mes lluvioso. Azul: mes muy lluvioso. Grisado Naranja: Núcleos pluviométricos.
Tabla N ${ }^{\circ}$ 3. Meses Iluviosos y Núcleos pluviométricos en Machagai. 1961-2005.

\begin{tabular}{|c|c|c|c|c|c|c|c|c|c|c|c|c|}
\hline AÑO & $E$ & $F$ & $M$ & $A$ & $M$ & J & $\mathrm{J}$ & A & $\mathrm{s}$ & 0 & $\mathrm{~N}$ & D \\
\hline 1961 & 65 & 229 & 179 & 83 & 95 & 20 & 0 & 23 & 65 & 70 & 167 & 206 \\
\hline 1962 & 123 & 56 & 162 & 214 & 75 & 0 & 33 & 22 & 12 & 118 & 64 & 58 \\
\hline 1963 & 175 & 170 & 115 & 173 & 46 & 15 & 32 & 0 & 18 & 49 & 130 & 47 \\
\hline 1964 & 17 & 130 & 82 & 223 & 39 & 5 & 10 & 35 & 100 & 23 & 74 & 148 \\
\hline 1965 & 43 & 210 & 56 & 250 & 58 & 7 & 18 & 29 & 54 & 182 & 295 & 346 \\
\hline 1966 & 362 & 2144 & 2860 & 118 & 83 & 102 & 6 & 3 & 55 & 29 & 197 & 23 \\
\hline 1967 & 444 & 250 & 201 & 35 & 59 & 25 & 22 & 31 & 55 & 25 & 114 & 37 \\
\hline 1968 & 186 & 68 & 53 & 35 & 28 & 18 & 44 & 73 & 5 & 120 & 188 & 61 \\
\hline 1969 & 198 & 176 & 38 & 60 & 29 & 7 & 33 & 40 & 23 & 180 & 26 & 40 \\
\hline 1970 & 95 & 126 & 61 & 49 & 51 & 10 & 24 & 37 & 133 & 94 & $\begin{array}{l}7 \\
\end{array}$ & 40 \\
\hline 1971 & 135 & 87 & 124 & 9 & 36 & 4 & 30 & 5 & 13 & 51 & 78 & 43 \\
\hline 1972 & 70 & 19 & 225 & 99 & 55 & 37 & 89 & 5 & 30 & 95 & 85 & 77 \\
\hline 1973 & 334 & 81 & 167 & 119 & 86 & 106 & 98 & 22 & 9 & 95 & 84 & 266 \\
\hline 1974 & 164 & 474 & 108 & 205 & 86 & 29 & 25 & 34 & 30 & 60 & 72 & 110 \\
\hline 1975 & 11 & 70 & 202 & 83 & 48 & 91 & 2 & 43 & 46 & 3 & 231 & 69 \\
\hline 1976 & 143 & 74 & 107 & 18 & 5 & 0 & 2 & 0 & 0 & 63 & 22 & 52 \\
\hline 1977 & 121 & 57 & 107 & 78 & 94 & 22 & 30 & 1 & 58 & 65 & 153 & 28 \\
\hline 1978 & 67 & 174 & 42 & 31 & 6 & 59 & 2 & 0 & 19 & 97 & 119 & 196 \\
\hline 1979 & 38 & 159 & 208 & 49 & 1 & 0 & 0 & 82 & 62 & 127 & 142 & 234 \\
\hline 1980 & 48 & 40 & 137 & 23 & 71 & 62 & 0 & 29 & 9 & 23 & 242 & 39 \\
\hline 1981 & 207 & 212 & 144 & 151 & 57 & 52 & 24 & 37 & 20 & 29 & 204 & 92 \\
\hline 1982 & 128 & 131 & 106 & 55 & 62 & 38 & 0 & 79 & 123 & 42 & 255 & 75 \\
\hline 1983 & 86 & 105 & 47 & 399 & 2408 & 0 & 26 & 0 & 0 & 4 & 68 & 66 \\
\hline 1984 & 80 & 10 & 352 & 132 & 32 & 43 & 0 & 30 & 82 & 237 & 177 & 15 \\
\hline 1985 & 48 & 101 & 79 & 478 & 62 & 0 & 9 & 46 & 78 & 27 & 19 & 104 \\
\hline 1986 & 18 & 121 & 433 & 244 & 26 & 86 & 25 & 7 & 66 & 184 & 181 & 39 \\
\hline 1987 & 127 & 89 & 71 & 174 & 100 & 8 & 40 & 5 & 0 & 80 & 134 & 188 \\
\hline 1988 & 215 & 89 & 183 & 206 & 2 & 10 & 6 & 0 & 10 & 11 & 62 & 142 \\
\hline 1989 & 103 & 64 & 260 & 176 & 2 & 17 & 18 & 83 & 15 & 73 & 66 & 249 \\
\hline 1990 & 149 & 106 & 64 & 268 & 33 & 33 & 67 & 20 & 95 & 251 & 62 & 144 \\
\hline 1991 & 127 & 159 & 50 & 336 & 2318 & 46 & 6 & 0 & 89 & 165 & 81 & 132 \\
\hline 1992 & 150 & 141 & 212 & 255 & 0 & 74 & 11 & 47 & 36 & 186 & 151 & 340 \\
\hline 1993 & 110 & 15 & 155 & 55 & 15 & 0 & 8 & 10 & 42 & 94 & 266 & 201 \\
\hline 1994 & 107 & 21214 & 166 & 478 & 235 & 78 & 15 & 40 & 0 & 1662 & 502 & 2312 \\
\hline 1995 & 342 & 141 & 148 & 58 & 63 & 2 & 3 & 31 & 38 & 77 & 83 & 52 \\
\hline 1996 & 274 & 384 & 1576 & 280 & 10 & 0 & 0 & 5 & 75 & 106 & 84 & 165 \\
\hline 1997 & 143 & -15. & 18 & 37 & 20 & 23 & 9 & 32 & 271 & 230 & 255 & 88 \\
\hline 1998 & 118,8 & 368 & 161 & 201 & 38 & 74 & 0 & 38 & 13 & 82 & 214 & 229 \\
\hline 1999 & 123 & 177 & 190 & 66 & 25 & 19 & 33 & 0 & 12 & 20 & 50 & 91 \\
\hline 2000 & 55 & 214 & 96 & 232 & 73 & 19 & 8 & 28 & 40 & 203 & 195 & 164 \\
\hline 2001 & 123 & 17 & 52 & 138 & 2 & 46 & 10 & 18 & 31 & 80 & 136 & 75 \\
\hline 2002 & 225 & 134 & 543 & 168 & 103 & 29 & 88 & 17 & 48 & 56 & 106 & 216 \\
\hline 2003 & 102 & 151 & 135 & 84 & 0 & 11 & 0 & 50 & 30 & 156 & 167 & 81 \\
\hline 2004 & 12 & 57 & 173 & 48 & 0 & 27 & 5 & 4 & 38 & 205 & 326 & 254 \\
\hline 2005 & 104 & 17 & 101 & 212 & 69 & 43 & 0 & 7 & 50 & 43 & 162 & 37 \\
\hline
\end{tabular}

Publicado en formato digital: Dr. Prof. Oscar Luis Pyszczek. DETECCIÓN DE PERIODOS HÚMEDOS EN LA PROVINCIA DEL CHACO (1961-2005). UNA APROXIMACION A LA IDENTIFICACIÓN DE NÚCLEOS PLUVIOMÉTRICOS. Revista Geográfica Digital. IGUNNE. Facultad de Humanidades. UNNE. Año 11. № 22. Julio - Diciembre. 2014. ISSN 1668-5180 Resistencia, Chaco.

En: http://hum.unne.edu.ar/revistas/geoweb/default.htm 
Celeste: mes lluvioso

Azul: mes muy lluvioso.

Grisado Naranja: Núcleos

pluviométricos.

Tabla $N^{\circ}$ 4. Meses lluviosos y Núcleos pluviométricos en San Bernardo. 1961-2005.

\begin{tabular}{|c|c|c|c|c|c|c|c|c|c|c|c|c|}
\hline AÑO & $E$ & $F$ & $M$ & A & $M$ & $\mathrm{~J}$ & $\mathrm{~J}$ & A & $\mathrm{s}$ & 0 & $\mathrm{~N}$ & $D$ \\
\hline 1961 & 21 & 215 & 2220 & 199 & 35 & 20 & 2 & 22 & 13 & 102 & 1110 & 172 \\
\hline 1962 & 158 & 68 & 85 & 135 & 12 & 7 & 28 & 30 & 0 & 69 & 54 & 70 \\
\hline 1963 & 123 & 153 & 15 & 59 & 20 & 0 & 22 & 0 & 128 & 85 & 75 & 157 \\
\hline 1964 & 28 & 1115 & 218 & 320 & 33 & 17 & 10 & 20 & 101 & 35 & 81 & 60 \\
\hline 1965 & 35 & 98 & 110 & 110 & 8 & 0 & 15 & 39 & 69 & 1238 & $48 \%$ & 253 \\
\hline 1966 & 2312 & 259 & 156 & 48 & 100 & 0 & 16 & 0 & 65 & 48 & 120 & 14 \\
\hline 1967 & 262 & 195 & 1118 & 40 & 85 & 19 & 0 & 20 & 45 & 39 & 65 & 43 \\
\hline 1968 & 212 & 75 & 46 & 94 & 18 & 40 & 45 & 64 & 0 & 45 & 70 & 24 \\
\hline 1969 & 176 & 243 & 54 & 87 & 45 & 0 & 33 & 0 & 97 & 170 & 26 & 99 \\
\hline 1970 & 120 & 92 & 78 & 38 & 1111 & 0 & 25 & 85 & 74 & 56 & 21 & 296 \\
\hline 1971 & 50 & 54 & 278 & 83 & 171 & 0 & 46 & 24 & 15 & 79 & 48 & 61 \\
\hline 1972 & 62 & 25 & 379 & 81 & 48 & 40 & 53 & 7 & 12 & 84 & 2144 & 206 \\
\hline 1973 & 259 & 34 & 266 & 149 & 6 & 87 & 64 & 19 & 8 & 82 & 102 & 77 \\
\hline 1974 & 128 & 332 & 46 & 201 & 115 & 4 & 22 & 18 & 13 & 34 & 9 & 22 \\
\hline 1975 & 35 & 88 & 376 & 112 & 50 & 46 & 2 & 26 & 21 & 60 & 191 & 49 \\
\hline 1976 & 155 & 68 & 263 & 8 & 6 & 3 & 7 & 7 & 24 & 83 & 78 & 92 \\
\hline 1977 & 176 & 101 & 299 & 79 & 51 & 6 & 15 & 34 & 46 & 75 & 151 & 97 \\
\hline 1978 & 80 & 167 & 22 & 34 & 3 & 12 & 0 & 0 & 41 & 96 & 158 & 90 \\
\hline 1979 & 150 & 176 & 84 & 71 & 0 & 0 & 57 & 24 & 48 & 74 & 50 & 243 \\
\hline 1980 & 37 & 158 & 95 & 197 & 118 & 38 & 3 & 0 & 45 & 77 & 184 & 86 \\
\hline 1981 & 289 & 245 & 166 & 124 & 121 & 17 & 15 & 27 & 7 & 47 & 101 & 111 \\
\hline 1982 & 151 & 85 & 102 & 107 & 5 & 40 & 16 & 1 & 182 & 56 & 114 & 198 \\
\hline 1983 & 159 & 128 & 90 & 393 & 84 & 1 & 48 & 5 & 12 & 18 & 116 & 15 \\
\hline 1984 & 233 & 259 & 539 & 452 & 41 & 28 & 5 & 12 & 58 & 0100 & 1332 & 123 \\
\hline 1985 & 124 & 284 & 070 & 306 & 75 & 25 & 12 & 8 & 57 & 78 & 78 & 136 \\
\hline 1986 & 50 & 91 & 428 & 248 & $5 \%$ & 59 & 82 & 14 & 251 & 88 & 162 & 70 \\
\hline 1987 & 150 & 56 & 73 & 102 & 89 & 2 & 33 & 19 & 58 & 26 & 239 & 120 \\
\hline 1988 & 120 & 47 & 119 & 84 & 3 & 13 & 17 & 0 & 14 & 34 & 46 & 101 \\
\hline 1989 & 33 & 50 & 245 & 530 & 4 & 16 & 56 & 17 & 19 & 54 & 43 & 220 \\
\hline 1990 & 100 & 38 & 79 & 223 & 59 & 18 & 15 & 0 & 32 & 244 & 53 & 97 \\
\hline 1991 & 119 & 55 & 186 & 255 & 295 & 7 & 14 & 12 & 92 & 48 & 40 & 100 \\
\hline 1992 & 75 & 145 & 86 & 142 & 1 & 0 & 38 & 68 & 11 & 2335 & 481 & 154 \\
\hline 1993 & 452 & 0 & 116 & 152 & 31 & 1 & 4 & 1 & 7 & 22 & 168 & 73 \\
\hline 1994 & 12 & 134 & 93 & 65 & 66 & 42 & 5 & 8 & 33 & 123 & 251 & 16 \\
\hline 1995 & 148 & 122 & 202 & 96 & 34 & 12 & 11 & 7 & 69 & 52 & 54 & 94 \\
\hline 1996 & 150 & 318 & 198 & 455 & 6 & 1 & 0 & 4 & 62 & 63 & 349 & 146 \\
\hline 1997 & 73 & 177 & 57 & 22 & 30 & 13 & 1 & 4 & 7 & 75 & 119 & 80 \\
\hline 1998 & 205 & 241 & 147 & 115 & 24 & 57 & 3 & 23 & 8 & 88 & 124 & 243 \\
\hline 1999 & 128 & 113 & 116 & 106 & 50 & 21 & 10 & 3 & 41 & 33 & 44 & 96 \\
\hline 2000 & 258 & 1115 & 171 & 91 & 34 & 28 & 4 & 25 & 50 & 212 & 152 & 105 \\
\hline 2001 & 132 & 164 & 102 & 141 & 4 & 72 & 3 & 30 & 40 & 475 & 169 & 126 \\
\hline 2002 & 2351 & 146 & 2224 & 258 & 23 & 4 & 101 & 1 & tor & 104 & 134 & 218 \\
\hline 2003 & 102 & 17 & 169 & 77 & 3 & 4 & 6 & 20 & 23 & 149 & 95 & 91 \\
\hline 2004 & 46 & 210 & 153 & 63 & 1 & 23 & 3 & 0 & 0 & 0 & 0 & 0 \\
\hline
\end{tabular}

Publicado en formato digital: Dr. Prof. Oscar Luis Pyszczek. DETECCION DE PERIODOS HÚMEDOS EN LA PROVINCIA DEL CHACO (1961-2005). UNA APROXIMACION A LA IDENTIFICACIÓN DE NÚCLEOS PLUVIOMÉTRICOS. Revista Geográfica Digital. IGUNNE. Facultad de Humanidades. UNNE. Año 11. № 22. Julio - Diciembre. 2014. ISSN 1668-5180 Resistencia, Chaco.

En: http://hum.unne.edu.ar/revistas/geoweb/default.htm 
Celeste: mes lluvioso. Azul: mes muy lluvioso. Grisado Naranja: Núcleos pluviométricos

Tabla No 5. Meses lluviosos y Núcleos pluviométricos en J.J. Castelli. 1961-2005.

\begin{tabular}{|c|c|c|c|c|c|c|c|c|c|c|c|c|}
\hline ANNO & $\bar{E}$ & $F$ & $M$ & $A$ & $M$ & J & J & $A$ & S & 0 & $\mathrm{~N}$ & D \\
\hline 1961 & 91 & 249 & 73 & 112 & 25 & 10 & 25 & 0 & 3 & 69 & 105 & 68 \\
\hline 1962 & 196 & 414 & 193 & 89 & 34 & 5 & 26 & 2 & 50 & 81 & 68 & 92 \\
\hline 1963 & 126 & 109 & 44 & 79 & 14 & 51 & 10 & 1 & 35 & 11 & 54 & 150 \\
\hline 1964 & 26 & 27 & 67 & 194 & 63 & 10 & 0 & 17 & 73 & 101 & 7 & 162 \\
\hline 1965 & 106 & 138 & 17 & 117 & 110 & 28 & 18 & 5 & 20 & 415 & 468 & 206 \\
\hline 1966 & 123 & 105 & 66 & 132 & 23 & 32 & 11 & 16 & 28 & 27 & 147 & 41 \\
\hline 1967 & 87 & 658 & 180 & 2328 & 30 & 0 & 91 & 33 & 34 & 26 & 119 & 58 \\
\hline 1968 & 155 & 192 & 150 & 31 & 41 & 33 & 32 & 28 & 38 & 108 & 58 & 139 \\
\hline 1969 & 219 & 104 & 34 & 106 & 129 & 1 & 15 & 20 & 85 & 58 & 50 & 85 \\
\hline 1970 & 87 & 106 & 125 & 60 & 24 & 16 & 13 & 49 & 47 & 88 & 84 & 0 \\
\hline 1971 & 122 & 91 & 168 & 53 & 86 & 19 & 47 & 16 & 24 & 59 & 24 & 80 \\
\hline 1972 & 167 & 15 & 188 & 89 & 49 & 19 & 83 & 24 & 13 & 72 & 84 & 27 \\
\hline 1973 & 32 & 35 & 68 & 89 & 33 & 120 & 45 & 3 & 19 & 33 & 137 & 71 \\
\hline 1974 & 92 & 43 & 105 & 107 & 80 & 6 & 15 & 33 & 20 & 78 & 22 & 136 \\
\hline 1975 & 45 & 48 & 331 & 458 & 441 & 34 & 4 & 37 & 17 & 22 & 117 & 87 \\
\hline 1976 & 143 & 28 & 92 & 11 & 7 & 4 & 0 & 6 & 40 & 84 & 24 & 36 \\
\hline 1977 & 235 & 58 & 148 & 84 & 60 & 3 & 25 & 49 & 12 & 46 & 62 & 57 \\
\hline 1978 & 204 & 152 & 30 & 20 & 40 & 0 & 0 & 0 & 44 & 78 & 185 & 1244 \\
\hline 1979 & 6 & 197 & 138 & 23 & 9 & 9 & 3 & 5 & 47 & 110 & 93 & 170 \\
\hline 1980 & 105 & 62 & 50 & 1918 & 135 & 95 & 0 & 14 & 40 & 58 & 8500 & 1442 \\
\hline 1981 & $62 x$ & 0 & 50 & 75 & 35 & 2 & 4 & 8 & 18 & 32 & 148 & 113 \\
\hline 1982 & 36 & 100 & 113 & 102 & 13 & 20 & 0 & 10 & 64 & 41 & 79 & 162 \\
\hline 1983 & 172 & 62 & 24 & 60 & 163 & 0 & 26 & 0 & 0 & 2 & 1115 & 70 \\
\hline 1984 & 111 & 20 & 77 & 97 & 62 & 35 & 0 & 2 & 13 & 156 & 127 & 30 \\
\hline 1985 & 59 & 248 & 23 & 302 & 2 & 9 & 5 & 5 & 151 & 206 & 18 & 104 \\
\hline 1986 & 7 & 62 & 415 & 257 & 69 & 54 & 9 & 0 & 75 & 49 & 245 & 424 \\
\hline 1987 & 238 & 50 & 7 & 38 & 17 & 5 & 62 & 40 & 20 & 96 & 112 & 66 \\
\hline 1988 & 70 & 48 & 179 & 101 & 2 & 13 & 0 & 0 & 0 & 35 & 86 & 184 \\
\hline 1989 & 91 & 37 & 217 & 158 & 15 & 20 & 10 & 50 & 8 & 10 & 108 & 84 \\
\hline 1990 & 60 & 132 & 5 & 131 & 16 & 50 & 18 & 15 & 87 & 55 & 31 & 90 \\
\hline 1991 & 34 & 163 & 2555 & 28 & 98 & 46 & 0 & 0 & 26 & 81 & 122 & 191 \\
\hline 1992 & 97 & 98 & 36 & 84 & 24 & 34 & 0 & 10 & 74 & 170 & 108 & 185 \\
\hline 1993 & 36 & 32 & 86 & 85 & 2 & 0 & 0 & 5 & 6 & 145 & 234 & 80 \\
\hline 1994 & 135 & 182 & 76 & 73 & 116 & 0 & 10 & 28 & 0 & 143 & 149 & 35 \\
\hline 1995 & 137 & 145 & 79 & 96 & 30 & 3 & 0 & 3 & 10 & 0 & 90 & 55 \\
\hline 1996 & 94 & 2153 & 194 & 1351 & 58 & 0 & 0 & 15 & 40 & 172 & 91 & 47 \\
\hline 1997 & 80 & 180 & 76 & 5 & 5 & 2 & 0 & 0 & 23 & 82 & 166 & 20 \\
\hline 1998 & 70 & 455 & 150 & 161 & 3 & 42 & 4 & 15 & 20 & 40 & 114 & 84 \\
\hline 1999 & 173 & 73 & 92 & 93 & 10 & 0 & 2 & 0 & 23 & 32 & 82 & 125 \\
\hline 2000 & 95 & 200 & 48 & 199 & 9 & 14 & 0 & 7 & 3 & 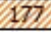 & 232 & 144 \\
\hline 2001 & 109 & 91 & 35 & 100 & 0 & 4 & 0 & 15 & 22 & 32 & 33 & 84 \\
\hline 2002 & 253 & 79 & 300 & 499. & 1227 & 11 & 29 & 3 & 58 & 35 & 179 & 28 \\
\hline 2003 & 262 & 43 & 143 & 95 & 0 & 7 & 0 & 45 & 24 & 49 & 34 & 218 \\
\hline 2004 & 57 & 0 & 204 & 26 & 0 & 0 & 10 & 0 & 12 & 39 & 134 & 184 \\
\hline 2005 & 52 & 14 & 40 & 138 & 65 & 49 & 24 & 0 & 5 & 15 & 142 & 63 \\
\hline
\end{tabular}

Publicado en formato digital: Dr. Prof. Oscar Luis Pyszczek. DETECCION DE PERIODOS HÚMEDOS EN LA PROVINCIA DEL CHACO (1961-2005). UNA APROXIMACION A LA IDENTIFICACIÓN DE NÚCLEOS PLUVIOMÉTRICOS. Revista Geográfica Digital. IGUNNE. Facultad de Humanidades. UNNE. Año 11. No 22. Julio - Diciembre. 2014. ISSN 1668-5180 Resistencia, Chaco.

En: http://hum.unne.edu.ar/revistas/geoweb/default.htm 
Celeste: mes lluvioso. Azul: mes muy lluvioso. Grisado Naranja: Núcleos pluviométricos.

Tabla No 6. Meses Iluviosos y Núcleos pluviométricos en Los Frentones. 1961-2005.

\begin{tabular}{|c|c|c|c|c|c|c|c|c|c|c|c|c|}
\hline ANNO & $\bar{E}$ & $F$ & $M$ & A & $M$ & J & J & $A$ & $s$ & 0 & $\mathrm{~N}$ & $D$ \\
\hline 1961 & 84 & 332 & 125 & 83 & 18 & 16 & 6 & 6 & 0 & 80 & 156 & 83 \\
\hline 1962 & 167 & 68 & 45 & 61 & 10 & 0 & 23 & 42 & 5 & 58 & 75 & 110 \\
\hline 1963 & 180 & 157 & 35 & 101 & 0 & 10 & 7 & 0 & 25 & 52 & 67 & 79 \\
\hline 1964 & 124 & 54 & 215 & 1571 & 40 & 125 & 200 & 0 & 70 & 65 & 20 & 139 \\
\hline 1965 & 45 & 144 & 55 & 41 & 15 & 0 & 15 & 0 & 18 & 0 & 1322 & 2556 \\
\hline 1966 & 214 & 256 & 155 & 33 & 66 & 0 & 0 & 0 & 59 & 44 & 130 & 11 \\
\hline 1967 & 200 & 138 & 87 & 45 & 56 & 5 & 30 & 34 & 50 & 26 & 34 & 62 \\
\hline 1968 & 184 & 59 & 68 & 67 & 15 & 49 & 25 & 65 & 42 & 52 & 89 & 191 \\
\hline 1969 & 187 & 186 & 32 & 67 & 47 & 8 & 25 & 0 & 16 & 46 & 18 & 98 \\
\hline 1970 & 85 & 47 & 209 & 52 & 73 & 12 & 32 & 25 & 33 & 55 & 37 & 109 \\
\hline 1971 & 94 & 141 & 148 & 67 & 65 & 0 & 140 & 56 & 15 & 84 & 40 & 44 \\
\hline 1972 & 61 & 51 & 137 & 111 & 0 & 10 & 35 & 0 & 16 & 0 & 122 & 81 \\
\hline 1973 & 313 & 68 & 110 & 177 & 28 & 92 & 19 & 5 & 0 & 15 & 103 & 55 \\
\hline 1974 & 121 & 235 & 169 & 32 & 119 & 0 & 16 & 12 & 30 & 18 & 30 & 108 \\
\hline 1975 & 116 & 59 & 204 & 103 & 87 & 41 & 1 & 10 & 0 & 35 & 103 & 85 \\
\hline 1976 & 121 & 113 & 96 & 0 & 0 & 0 & 0 & 0 & 24 & 45 & 95 & 83 \\
\hline 1977 & 53 & 90 & 248 & 78 & 33 & 3 & 25 & 26 & 12 & 8 & 134 & 245 \\
\hline 1978 & 89 & 165 & 46 & 12 & 0 & 0 & 0 & 0 & 32 & 116 & 82 & 63 \\
\hline 1979 & 35 & 125 & 202 & 74 & 0 & 28 & 4 & 0 & 47 & 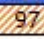 & 156 & 248 \\
\hline 1980 & 127 & 50 & 438 & 240 & 1804 & 180 & 0 & 0 & 13 & 35 & 180 & 125 \\
\hline 1981 & 192 & 126 & 75 & 70 & 93 & 0 & 0 & 0 & 7 & 10 & 158 & 100 \\
\hline 1982 & 147 & 128 & 181 & 28 & 10 & 0 & 0 & 0 & 73 & 32 & 61 & 193 \\
\hline 1983 & 80 & 146 & 100 & 219 & 67 & 0 & 33 & 0 & 0 & 60 & 184 & 89 \\
\hline 1984 & 211 & 171 & 50 & 122 & 15 & 15 & 0 & 3 & 55 & 90 & 126 & 23 \\
\hline 1985 & 101 & 120 & 110 & 152 & 4 & 25 & 10 & 52 & 22 & 67 & 154 & 70 \\
\hline 1986 & 24 & 153 & 311 & 1621 & 45 & 46 & 23 & 45 & 40 & 250 & 234 & 95 \\
\hline 1987 & 182 & 22 & 23 & 103 & 45 & 8 & 43 & 8 & 0 & 66 & 234 & 78 \\
\hline 1988 & 129 & 15 & 10 & 24 & 0 & 0 & 9 & 0 & 3 & 10 & 81 & 60 \\
\hline 1989 & 0 & 0 & 125 & 183 & 15 & 0 & 15 & 10 & 26 & 0 & 50 & 135 \\
\hline 1990 & 75 & 65 & 18 & 154 & 57 & 31 & 30 & 0 & 3 & 104 & 107 & 98 \\
\hline 1991 & 120 & 200 & 21216 & 85 & 110 & 9 & 0 & 0 & 0 & 20 & 43 & 0 \\
\hline 1992 & 161 & 126 & 18 & 110 & 18 & 10 & 0 & 24 & 48 & 249 & 165 & 218 \\
\hline 1993 & 90 & 25 & 120 & 54 & 0 & 0 & 0 & 0 & 0 & 65 & 194 & 133 \\
\hline 1994 & 121 & 90 & 91 & 24 & 73 & 8 & 0 & 12 & 72 & 103 & 100 & 90 \\
\hline 1995 & 263 & 131 & 120 & 70 & 128 & 10 & 0 & 0 & 4 & 55 & 64 & 135 \\
\hline 1996 & 57 & 178 & (197) & 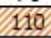 & 11 & 0 & 0 & 0 & 46 & 161 & 54 & 234 \\
\hline 1997 & 101 & 95 & 110 & 19 & 0 & 5 & 0 & 0 & 6 & 84 & 69 & 108 \\
\hline 1998 & 129 & 358 & 106 & 191 & 2 & 9 & 8 & 20 & 10 & 33 & 49 & 102 \\
\hline 1999 & 84 & 140 & 129 & 60 & 7 & 0 & 7 & 0 & 45 & 30 & 37 & 74 \\
\hline 2000 & 138 & 184 & 114 & 216 & 17 & 0 & 3 & 0 & 6 & 150 & 99 & 89 \\
\hline 2001 & 114 & 101 & 84 & 43 & 0 & 31 & 0 & 13 & 20 & 33 & 84 & 56 \\
\hline 2002 & 255 & 194 & 300 & 1447 & 2 & 2 & 28 & 0 & 17 & 54 & 74 & 143 \\
\hline 2003 & 130 & 28 & 311 & 45 & 0 & 12 & 0 & 22 & 24 & 25 & 32 & 68 \\
\hline 2004 & 61 & 0 & 133 & 2 & 0 & 4 & 0 & 0 & 0 & 34 & 25 & 136 \\
\hline 2005 & 0 & 21 & 36 & 128 & 4 & 11 & 0 & 0 & 25 & 0 & 308 & 73 \\
\hline
\end{tabular}

Publicado en formato digital: Dr. Prof. Oscar Luis Pyszczek. DETECCION DE PERíODOS HÚMEDOS EN LA PROVINCIA DEL CHACO (1961-2005), UNA APROXIMACION A LA IDENTIFICACIÓN DE NÚCLEOS PLUVIOMÉTRICOS. Revista Geográfica Digital. IGUNNE. Facultad de Humanidades. UNNE. Año 11. № 22. Julio - Diciembre. 2014. ISSN 1668-5180 Resistencia, Chaco.

En: http://hum.unne.edu.ar/revistas/geoweb/default.htm 
Revista Geográfica Digital. IGUNNE. Facultad de Humanidades. UNNE. Año 11. № 22. Julio - Diciembre 2014. ISSN 1668-5180 Resistencia, Chaco

\section{Conclusiones.}

El desarrollo de la investigación acerca del comportamiento y distribución de las precipitaciones, en la Provincia del Chaco, durante el período 1961-2005; permite destacar una serie de situaciones que a criterio propio resultan importantes:

* La variabilidad registrada entre los datos contenidos en la serie estadística, se incrementan hacia el oeste de la provincia.

* Los menores montos pluviométricos pueden registrarse antes o después de los picos pluviales, lo que permite pensar sobre una compensación pluvial local.

- Existen años y/o períodos donde puede observarse una sincronía entre los valores pluviométricos de los puestos estudiados, tanto en bajos montos pluviométricos (1989), como así también en montos considerables (1965-68).

- Las regularidades encontradas entre los datos de los puestos pluviométricos tomados como referencia, denotan controles atmosféricos que actúan a escala Regional y Global.

La determinación e identificación en la serie estadística de los meses y períodos húmedos ha implicado trabajar mediante una convergencia de metodologías que conlleve a la individualización de montos pluviométricos superiores o inferiores a lo normal.

La distribución de los meses lluviosos y muy lluviosos en las series estadísticas correspondientes a cada una de los puestos pluviométricos, presentan en general una correspondencia con los años donde se han producido eventos hídricos de relevancia como las inundaciones de 1982-83; 1998.

la extensión de los núcleos pluviométricos son mayores en los meses de verano y menos prolongados en el otoño.

Ahora bien la existencia de meses lluviosos o muy lluviosos no determina la ocurrencia de los períodos húmedos, los cuales se relacionan con la intensidad y prolongación de tales meses lluviosos o muy lluviosos.

La variabilidad que presentan las precipitaciones, hace difícil la detección de una tendencia firme en los datos. No obstante es posible detectar oscilaciones, que por ejemplo posibilitaron la expansión de la frontera agrícola en el oeste de la provincia, multiplicándose, los campos en donde actualmente se cultiva soja.

Los déficits y excesos pluviométricos, ejercen una acción muy negativa en las actividades productivas primarias, pero son situaciones necesarias para la regulación o compensación del sistema pluvial y climático. Esta compensación del sistema es visible en los datos estadísticos de las series antes tratadas, y justamente por ello es que la fuerte sequía vivida en los últimos años en la provincia no necesariamente está vinculada al proceso de Cambio Climático, tan en boga en los últimos tiempos o bien para afirmar si existe alguna relación, habría que esperar varios años más.

Finalmente es necesario recalcar la necesidad de contar con registros meteorológicos de calidad de manera tal que las conclusiones de las investigaciones llevadas a cabo con los mismos sean significativas y permitan prever con anticipación, circunstancias atmosféricas que podrían afectar a las poblaciones.

\section{Bibliografía.}

ALBERTO, J. (2006). El Chaco Oriental y sus fisonomías vegetales. en: Revista Geográfica Digital. Enero-Junio 2006. Instituto de Geografía (IGUNNE). Facultad de Humanidades - UNNE.

BARRY, R. y CHORLEY, R. (1972). Atmósfera, tiempo y clima. Barcelona, Omega.

BRUNIARD, Enrique (1978). El Gran Chaco Argentino (ensayo de interpretación geográfica). En: GEOGRAFICA 4. Revista del Instituto de Geografía. Resistencia, Facultad de Humanidades, UNNE.

BRUNIARD, Enrique (1990). El clima de las planicies del norte argentino (ensayo metodológico de geografía de los climas). Resistencia, Facultad de Humanidades, UNNE.

Publicado en formato digital: Dr. Prof. Oscar Luis Pyszczek. DETECCIÓN DE PERíodos HÚMEDOS EN LA PROVINCIA DEL CHACO (1961-2005). UNA APROXIMACIÓN A LA IDENTIFICACIÓN DE NÚCLEOS PLUVIOMÉTRICOS. Revista Geográfica Digital. IGUNNE. Facultad de Humanidades. UNNE. Año 11. Nº 22. Julio - Diciembre. 2014. ISSN 1668-5180 Resistencia, Chaco.

En: http://hum.unne.edu.ar/revistas/geoweb/default.htm 
Revista Geográfica Digital. IGUNNE. Facultad de Humanidades. UNNE. Año 11. № 22. Julio - Diciembre 2014. ISSN 1668-5180 Resistencia, Chaco

BRUNIARD, Enrique (1992). El ámbito subtropical en la República Argentina (Climatología dinámica y límites climáticos). En: Revista Estudios Geográficos. Madrid, Instituto de Economía y Geografía Aplicada, $\mathrm{N}^{\circ} 208$.

BRUNIARD, Enrique (1992). Climatología. Procesos y tipos climáticos. Buenos Aires, CEYNE.

BURGOS, Juan (1970). El clima de la región Noreste de la República Argentina en relación con la vegetación natural y el suelo. En: Boletín de la Sociedad Argentina de Botánica. Buenos Aires, Sociedad Argentina de Botánica, vol. XI, pp. 37-102.

CARVALHO, L., JONES, C. y LIEBMANN, B. (2002). Extreme Precipitation Events in Southeastern South America and Large-Scale Convective Patterns in the South Atlantic Convergence Zone. Journal of Climate, Vol 15, No 17 , pp. 2377-2394

CUADRAT, José María y PITA, María Fernanda (1999). Climatología. Madrid, Cátedra.

CHIOZZA, E. y GONZALEZ VAN DOMSELAAR, C. (1958). Clima. En: La Argentina, Suma de Geografía. Buenos Aires, Peuser, t. II.

EASTERLING, D. R.; EVANS, J. L.; GROISMAN, Y.; KARL, T. R.; KUNKEL, K. E. and AMBENJE, P. (2000). Observed Variability and Trends in Extreme Climate Events: A brief review. Bulletin of the American Meteorological Society, 81, pp. 417-425.

FERNANDEZ GARCIA, F. (1996). Manual de Climatología Aplicada. Clima, Medio Ambiente y Planificación. Madrid, Síntesis.

GALMARINI, A. y RAFFO DEL CAMPO, J. (1964). Rasgos fundamentales que caracterizan el clima de la Región Chaqueña. Buenos Aires, Consejo Nacional de Desarrollo (CONADE).

GRIMM, A., BARROS, V. y DOYLE, M. (2000). Climate Variability in Southern South America Associated with El Niño and La Niña Events. Journal of Climate, Vol. 13, № 1, pp. 35/58.

KARL, T. R. and EASTERLING, D. R. (1999). Climate extremes: Selected review and future research directions. Climatic Change, № 42, pp. 309-325.

KNOCHE, Walter y BORZACOV, Vladimir (1947). Clima de la República Argentina. En: Geografía de la República Argentina. Buenos Aires, Imp. Coni, ts. V y VI.

KIDSON, J. (1988). Interannual Variations in the Southern Hemisphere Circulation. Journal of Climate, Vol. 1, No 12, pp. 1177/1198.

KILADIS, George y DIAZ, Henry. (1989). Global Climatic Anomalies Associated with Extremes in the Southern Oscillation. Journal of Climate, Vol. 2, No 9, pp. 1069/1090.

MECHOSO, C. y PEREZ IRIBARREN, G. (1992). Streamflow in Southeastern South America and the Southern Oscillation. Journal of Climate, Vol. 5, № 12, pp. 1535/1549.

PÉREZ, MARÍA EMILIA (2009) "El comportamiento temporo espacial de los montos anuales de precipitación en el nordeste argentino - 1931/2005". Revista Geográfica Digital. IGUNNE. Facultad de Humanidades. UNNE. Año $6 \mathrm{n}^{\circ} 11$ enero- junio de 2009. Chaco.

PÉREZ, MARÍA EMILIA (2009) "el comportamiento temporal y espacial de los montos estacionales de precipitación en el nordeste argentino - 1931/2005". Revista Geográfica Digital. IGUNNE. Facultad de Humanidades. UNNE. Año $6 \mathrm{n}^{\circ} 12$ julio-diciembre de 2009.

PÉREZ, MARÍA EMILIA y GÓMEZ C. (2011) "La variabilidad pluviométrica en la provincia del chaco durante el período 1955 - 2009" ". Revista Geográfica Digital. IGUNNE. Facultad de Humanidades. UNNE. Año $6 n^{\circ} 12$ julio-diciembre de 2011.

PETTERSSEN, Sverr (1976). Introducción a la Meteorología. Madrid, Espasa Calpe.

PROHASKA, F. (1952). Regímenes estacionales de precipitación de Sudamérica y mares vecinos (desde $15^{\circ} \mathrm{S}$ hasta Antártida. En: Revista METEOROS. Buenos Aires, Servicio Meteorológico Nacional, , $\mathrm{Ns}^{\circ} 1-2$.

RODRIGUEZ Y CHEBEZ (2003) Patrimonio natural. Buenos Aires. Disponible en: http://www.patrimonionatural.com/HTML/provincias/chaco/chacopn/relieve.asp

WOLCKEN, K. (1954). Algunos aspectos sinópticos de la lluvia en la Argentina. En: Revista METEOROS. Buenos Aires, Servicio Meteorológico Nacional, № 4.

Publicado en formato digital: Dr. Prof. Oscar Luis Pyszczek. DETECCIÓN DE PERíodos HÚMEDOS EN LA PROVINCIA DEL CHACO (1961-2005). UNA APROXIMACIÓN A LA IDENTIFICACIÓN DE NÚCLEOS PLUVIOMÉtRICOS. Revista Geográfica Digital. IGUNNE. Facultad de Humanidades. UNNE. Año 11. N 22. Julio - Diciembre. 2014. ISSN 1668-5180 Resistencia, Chaco.

En: http://hum.unne.edu.ar/revistas/geoweb/default.htm 